\title{
Junctions in human health and inherited disease
}

\author{
Spiro Getsios ${ }^{1} \cdot$ David P. Kelsell $^{2} \cdot$ Andy Forge $^{3}$
}

Received: 4 March 2015 / Accepted: 5 March 2015 /Published online: 11 April 2015

(C) Springer-Verlag Berlin Heidelberg 2015

The ability of cells to adhere to and communicate with other cells and with the extracellular environment is crucial for morphogenesis and the maintenance of tissue integrity. A cell can adhere directly to another cell (cell-cell adhesion) or to components of the surrounding extracellular matrix (cell-matrix adhesion). These two basic types of interactions permit the integration of cells into distinct tissues and the transfer of information among cells and with their environment. In epithelia, the three major classes of cell junctions are the anchoring junctions (including adherens junctions, desmosomes, hemidesmosomes and focal adhesions), tight junctions and gap junctions. These cell junctions are dysregulated in many human disorders either by inherited gene mutation and/or during disease pathogenesis, examples of which form the basis of this Cell and Tissue Research Special Issue: Junctions in Health and Disease.

Desmosomes and hemidesmosomes serve as multi-protein complexes that link the intermediate filament-based cytoskeleton with the plasma membrane at anchorage points to adjacent cells or the underlying basement membrane, respectively. The immaculate ultrastructural organization and biochemical resilience of these junctions evident in tissues that bear the brunt of the body's mechanical load give the impression that

Spiro Getsios

s-getsios@northwestern.edu

1 Department of Dermatology, Northwestern University Feinberg School of Medicine, 303 E Chicago Avenue, Ward 9-132, Chicago IL 60611, USA

2 Centre for Cell Biology and Cutaneous Research, The Blizard Institute, Barts and The London School of Medicine and Dentistry, Queen Mary University of London, London E1 2AT, UK

3 Ear Institute, University College London, London WC1X 8EE, UK desmosomes and hemidesmosomes act as relatively inert spot welds. However, these Titans of cell adhesion have revealed themselves to be rather vulnerable targets of inherited, acquired and infectious diseases. Live cell and intravital imaging studies have shifted our perspective concerning the static nature of these junctions, which are now seen as reasonably malleable membrane complexes that respond to changes in the microenvironment with the dynamic exchange of many of their molecular components that move into distinct cytoplasmic compartments and, in some cases, the nucleus. Moreover, the singular role of desmosomes and hemidesmosomes as adhesive organelles has given way to a new appreciation of these junctions as integrators of signalling pathways controlling cell proliferation, motility, differentiation and survival.

Desmosomes have long been recognized as having roles in maintaining skin integrity and have more recently gained attention as targets not only of dermatological disorders but also of heart disease. An article by Stahley and Kowalczyk (2015) details the prominent function of desmosomes as adhesive organelles by reviewing the way that autoantibodies and bacterial toxins target these junctions to cause skin blistering diseases. Samuelov and Sprecher (2015) highlight the impact of an increasingly long list of inherited genetic mutations in desmosome proteins leading to more than the simple loss of cell-cell adhesion in skin, hair and heart. Among the many phenotypic manifestations of desmosome deficiency, they illustrate an unexpected link between these junctions and inflammation reflecting their abundance in epithelial linings and possibly a direct role in the modulation of skin barrier function. Desmosomes undergo a natural maturation process into corneodesmosomes in the stratum corneum of dry epithelia; when this process is dysregulated, it has a profound impact on barrier formation and disease as summarized in separate articles by Ishida-Yamamoto and Igawa 
(2015) and Haftek (2015). Finally, Calore et al. (2015) highlight the role of desmosomes as incorporated units of intercalated disks in cardiomyocytes with mutations in these genes frequently having negative consequences on related junctional proteins including the gap junction protein $\mathrm{Cx} 43$ (also see Lambiase and Tinker 2015) leading to arrhythmogenic cardiomyopathy.

An article by Broussard et al. (2015) provides a refresher on the best known functions of the major desmosome proteins and an update on the way that such molecular components also play important roles outside of these junctions by participating in a diverse array of signalling pathways regulating various cell behaviours. In order for desmosomes to control these complex adhesion-signalling networks that maintain tissue integrity and homeostasis in organs such as the skin, their expression needs to be tightly regulated. Ferone et al. (2015) focus on these aspects of desmosome regulation by highlighting the role of $\mathrm{p} 63$, a master regulator of epidermal differentiation and target of several inherited skin disorders, in this process.

Hemidesmosomes are specialized anchorage points for basal keratinocytes in the skin and are constructed via a series of hierarchically ordered protein-protein interactions that undergo extensive posttranslational modification to build connections with intermediate filaments in the cytoplasm and the laminin-rich basement membrane in the extracellular space, as detailed in the article by Walko et al. (2015). Turcan and Jonkman (2015) review the impact of inherited or autoimmune disorders targeting hemidesmosomes and leading to a spectrum of relatively mild to more severe blistering disorders often associated with inflammation. An article by Kenny and Connelly (2015) emphasizes the dynamic nature of these hemidesmosomes and other integrin-based cell-matrix adhesion complexes in epithelial cells; these structures play a key role in sensing and responding to mechanical cues in the extracellular microenvironment during tissue repair, regeneration and homeostasis.

The impressive molecular organization of hemidesmosomes and desmosomes requires a connection to the intermediate filament network system. In epithelial cells, specific type I and type II keratins serve as the major intermediate filament pair that interacts with these cell-cell and cell-matrix junctions via their respective cytolinker proteins. Inherited mutations in a number of keratin genes can lead to distinct skin diseases as reviewed by Knöbel et al. (2015). The authors also discuss approaches to normalize skin epithelium in such patients by targeting mutant keratin genes using short interfering RNA technologies. Intermediate filaments in skeletal and cardiac muscle are composed of desmin, whereas neurons employ neurofilaments for this purpose. Hnia et al. (2015) review the impact of desmin deficiency for skeletal and cardiac myopathies with insight into structural and signalling roles mediated by desmin filaments. In a similar vein, Gentil et al.
(2015) highlight the dynamic nature of neurofilaments and their impact on an array of neurological syndromes. Chojnowski et al. (2015) summarize the emerging role of A- and B-type lamins in maintaining nuclear architecture and organization with a profound impact on cell differentiation and ageing in diseases collectively known as laminopathies.

Gap junctions provide for direct communication between cells and for the transfer of ions and metabolites to maintain tissue homeostasis. Connexins are proteins that can form gap junction channels and mutations in specific connexin genes are associated with a variety of disorders. Gap junctions are composed of aggregates of channels through the membrane of one cell (a connexon or "hemichannel") in exact register with connexons in the apposed cell thereby forming continuous conduits that enable direct intercellular transfer. Each connexon is formed of six connexin subunits. The different connexins confer differing permeability and regulatory properties upon the channels. Channel properties are also influenced by whether all the connexins of an individual connexon are of the same or different sub-type (homomeric or heteromeric connexons/hemichannels) and whether the connexin composition of the hemichannel of one cell is the same or different from that with which it is paired in the opposing cell (homotypic or heterotypic gap junctions).

Recessive mutations in the GJB2 gene encoding connexin (Cx)26 are the most common cause of inherited hearing loss. Additionally, mutations in GJB6 encoding Cx30 can also result in hearing impairment. Jagger and Forge (2015) argue that, whereas evidence exists that the gap junctions in the cochlea composed of these two connexins provide a pathway for direct cell to cell passage of $\mathrm{K}^{+}$ions to maintain the ionic environment necessary for sound perception and sensory cell survival, channels of almost any composition could allow $\mathrm{K}^{+}$ transfer. Thus, the authors suggest that other connexin/gap junction roles might be affected by GJB2 or GJB6 mutation.

Although recessive $G J B 2$ or $G J B 6$ mutations affect only the cochlea resulting in "non-syndromic" deafness, specific dominant mutations in these two genes can also affect tissues in other organs, most commonly the skin in which Cx26 and $\mathrm{Cx} 30$ are also expressed. In addition, mutations in two other $\beta$-class connexins, namely $\mathrm{Cx} 31$ (GJB3) and $\mathrm{Cx} 30.3$ (GJB4), can also affect just the skin underlying the hyperproliferation skin disease called erythrokeratoderma. The role of $\beta$ connexins in maintaining homeostasis in skin and the manner in which this is affected by particular mutations is discussed by Martin and van Steensel (2015). Evidence is available that some mutations affect hemichannel properties such that, in some cases, they are inappropriately open thus providing channels between the cell interior and the extracellular environment. Therapeutic targeting of these channels is suggested as a means to treat inflammatory skin disorders. Another $\beta$ class connexin, Cx32, encoded by GJB1, is widely expressed by myelinating glial cells of the peripheral and central nervous 
systems. It is thought to form the channels in gap junctions between layers of the myelin sheaths around an axon to provide a path for metabolites from the cell body of the glial cell to each myelin layer. A large number of mutations in GJB1 have been associated with the neuropathies that characterize X-linked Charcot-Marie-Tooth (CMTX) disease. Here, Abrams and Freidin (2015) discuss the potential roles of $\mathrm{Cx} 32$ in the peripheral and central nervous systems and explain the way that the correlation of the genetics of the disease with the phenotype in patients combined with the targeted ablation of gjbl in mice is beginning to elucidate some of the bases of the functional defects observed.

Defects in connexins that result in aberrant intercellular communication are perhaps most particularly demonstrated in the heart. The most ubiquitous connexin expressed in the body is $\mathrm{Cx} 43$, the "founding member" of the $\alpha$-class of connexins. In the heart, gap junctions composed of $\mathrm{Cx} 43$, $\mathrm{Cx} 45$ and other $\alpha$-connexins provide for the electrical coupling between cardiomyocytes essential for the electrical conduction that maintains cardiac rhythms. Lambiase and Tinker (2015) explain the way that abnormal connexin function results in a variety of heart diseases. Connexin and gap junctional dysfunction can also affect cell proliferation, not only in skin as discussed by Martin and van Steensel (2015) but also with a range of other proliferative disorders including cancer as described by Aasen (2015). These effects can arise from defects in intercellular communication, signalling via hemichannels or non-junctional effects through interactions of connexins with other molecular signalling systems. Kelly et al. (2015) discuss the range of molecular mechanisms associated with various mutations in connexins and the way that these can provide the basis for finding appropriate therapies.

Unlike gap junctions, tight junctions are composed of products of several different gene families, each of which can have several members. The primary transmembrane proteins involved are claudins, occludins and tricellulin, together with certain peripheral proteins such as ZO-1 and ZO-2, some of which interact with actin. The tight junction acts as a permeability barrier in which proteins are organized in continuous strands in the plasma membranes around the necks of each adjacent cell thereby occluding the intercellular space but, as Brandner and Schulzke (2015) describe, the tightness of the barrier is affected by the precise composition of the junction, varies with tissue type and can be regulated to allow the passage of certain molecules or ions depending on the local environmental conditions. Adherens junctions act primarily to hold adjacent cells together through interactions between the extracellular domains of the transmembrane cadherin proteins of the adjacent cells. The intracellular domain of the cadherin is associated with catenin family members, which in turn interact with actin that forms an anchoring cytoskeleton at the site of the junction. The protein complexes localized at the adherens junction can also activate intracellular pathways to regulate the cytoskeleton or transcription in response to external or internal signals. Disruption of the adherens junctions or defects in the associated proteins are associated with a variety of diseases including inflammatory bowel disease (Mehta et al. 2015), disorders of the skin and hair (Samuelov et al. 2015) and cancer (Vite et al. 2015).

In summary, cell junctions are critical in normal human development and health. The disease mechanisms underlying the myriad of cell-junction-associated disorders still require further investigations. For example, the inter-relationship between the various cell junction molecules and their role in cell signalling and communication are current areas of exploration.

\section{References}

Aasen T (2015) Connexins: junctional and non-junctional modulators of proliferation. Cell Tissue Res. doi:10.1007/s00441-014-2078-3

Abrams CK, Freidin M (2015) GJB1-associated X-linked CharcotMarie-Tooth disease, a disorder affecting the central and peripheral nervous systems. Cell Tissue Res. doi:10.1007/s00441-014-2014-6

Brandner JM, Schulzke JD (2015) Hereditary barrier-related diseases involving the tight junction: lessons from skin and intestine. Cell Tissue Res. doi:10.1007/s00441-014-2096-1

Broussard JA, Getsios S, Green KJ (2015) Desmosome regulation and signaling in disease. Cell Tissue Res. doi:10.1007/s00441-0152136-5

Calore M, Lorenzon A, De Bortoli M, Poloni G, Rampazzo A (2015) Arrhythmogenic cardiomyopathy: a disease of intercalated discs. Cell Tissue Res. doi:10.1007/s00441-014-2015-5

Chojnowski A, Ong PF, Dreesen O (2015) Nuclear lamina remodelling and its implications for human disease. Cell Tissue Res. doi:10. 1007/s00441-014-2069-4

Ferone G, Mollo MR, Missero C (2015) Epidermal cell junctions and their regulation by p63 in health and disease. Cell Tissue Res. doi: 10.1007/s00441-014-2108-1

Gentil BJ, Tibshirani M, Durham HD (2015) Neurofilament dynamics and involvement in neurological disorders. Cell Tissue Res. doi:10. 1007/s00441-014-2082-7

Haftek M (2015) Epidermal barrier disorders and corneodesmosome defects. Cell Tissue Res. doi:10.1007/s00441-014-2019-1

Hnia K, Ramspacher C, Vermot J, Laporte J (2015) Desmin in muscle and associated diseases: beyond the structural function. Cell Tissue Res. doi:10.1007/s00441-014-2016-4

Ishida-Yamamoto A, Igawa S (2015) The biology and regulation of corneodesmosomes. Cell Tissue Res. doi:10.1007/s00441-0142037-z

Jagger DJ, Forge A (2015) Connexins and gap junctions in the inner ear-it's not just about $\mathrm{K}^{+}$recycling. Cell Tissue Res. doi:10. 1007/s00441-014-2029-z

Kelly JJ, Simek J, Laird DW (2015) Mechanisms linking connexin mutations to human diseases. Cell Tissue Res. doi:10.1007/s00441014-2024-4

Kenny FN, Connelly JT (2015) Integrin-mediated adhesion and mechano-sensing in cutaneous wound healing. Cell Tissue Res. doi:10.1007/s00441-014-2064-9

Knöbel M, O’Toole EA, Smith FJD (2015) Keratins and skin disease. Cell Tissue Res. doi:10.1007/s00441-014-2105-4 
Lambiase PD, Tinker A (2015) Connexins in the heart. Cell Tissue Res. doi:10.1007/s00441-014-2020-8

Martin PEM, van Steensel M (2015) Connexins and skin disease: insights into the role of beta connexins in skin homeostasis. Cell Tissue Res. doi:10.1007/s00441-014-2094-3

Mehta S, Nijhuis A, Kumagai T, Lindsay J, Silver A (2015) Defects in the adherens junction complex (E-cadherin/ $\beta$-catenin) in inflammatory bowel disease. Cell Tissue Res. doi:10.1007/s00441-014-1994-6

Samuelov L, Sprecher E (2015) Inherited desmosomal disorders. Cell Tissue Res. doi:10.1007/s00441-014-2062-y

Samuelov L, Sprecher E, Paus R (2015) The role of P-cadherin in skin biology and skin pathology: lessons from the hair follicle. Cell Tissue Res. doi:10.1007/s00441-015-2114-y
Stahley SN, Kowalczyk AP (2015) Desmosomes in acquired disease. Cell Tissue Res. doi:10.1007/s00441-015-2155-2

Turcan I, Jonkman MF (2015) Blistering disease: insight from the hemidesmosome and other components of the dermalepidermal junction. Cell Tissue Res. doi:10.1007/s00441014-2021-7

Vite A, Li J, Radice GL (2015) New functions for alpha-catenins in health and disease: from cancer to heart regeneration. Cell Tissue Res. doi: 10.1007/s00441-015-2123-x

Walko G, Castañón MJ, Wiche G (2015) Molecular architecture and function of the hemidesmosome. Cell Tissue Res. doi:10.1007/ s00441-014-2061-z 